PROCEEDINGS OF THE

AMERICAN MATHEMATICAL SOCIETY

Volume 140, Number 4, April 2012, Pages 1331-1341

S 0002-9939(2011)10992-4

Article electronically published on August 3, 2011

\title{
PERIODIC SOLUTIONS OF RADIALLY SYMMETRIC PERTURBATIONS OF NEWTONIAN SYSTEMS
}

\author{
ALESSANDRO FONDA AND RODICA TOADER
}

(Communicated by Yingei Yi)

\begin{abstract}
The classical Newton equation for the motion of a body in a gravitational central field is here modified in order to include periodic central forces. We prove that infinitely many periodic solutions still exist in this case. These solutions have periods which are large integer multiples of the period of the forcing and rotate exactly once around the origin in their period time.
\end{abstract}

\section{INTRODUCTION}

Newton's equation for the motion of a planet around the sun is of the type

$$
\ddot{x}=-c \frac{x}{|x|^{3}},
$$

where $c>0$ is a constant depending on the mass of the attracting body. As it is well known, this equation gives rise to a variety of periodic solutions having elliptical planar orbits.

We would like to investigate what happens with these periodic orbits when the given force is supposed to vary periodically in time, with some period $T>0$. For example, the positive constant $c$ could be replaced by a periodic function $c(t)$, and an external periodic central force could be added, as well.

Let us consider, e.g., the equation

$$
\ddot{x}=-c(t) \frac{x}{|x|^{\gamma}}+e(t) \frac{x}{|x|},
$$

where $c(t)$ and $e(t)$ are two $T$-periodic functions, with $c(t)$ positive. Let us denote by

$$
\bar{e}=\frac{1}{T} \int_{0}^{T} e(t) d t
$$

the mean value of $e(t)$. In $[\underline{5}$, we have already proved that if $1<\gamma<4$ and $\bar{e} \leq 0$, then equation (11) has infinitely many periodic solutions, whose periods are large multiples of $T$, rotating around the origin on large-amplitude orbits. In [7, the above result was refined by removing the restriction that $\gamma<4$.

It should be noticed that, besides the periodic solutions, a whole family of quasiperiodic solutions was detected in [5, 7]. Indeed, all those solutions have a $T$ periodic oscillating radial component, while their mean angular velocity covers a

Received by the editors November 30, 2009 and, in revised form, January 4, 2011.

2010 Mathematics Subject Classification. Primary 34C25.

Key words and phrases. Periodic solutions, Newton's equation, nonlinear dynamics. 
whole interval of positive values, among which one can find the sub-multiples of $2 \pi$ which correspond to the periodic (subharmonic) solutions.

In this paper we would like to investigate the complementary case when $\bar{e}>0$. As we have already proved in [5], in this case solutions with large-amplitude orbits cannot be periodic. We therefore look for periodic solutions with smaller amplitude. We will show that if $\gamma \geq 2$, even in this case there are infinitely many periodic solutions of (1). Hence, combining this result with the ones in [5, 17, we can finally state an existence theorem for periodic solutions of (1), valid for any choice of the $T$-periodic forcing $e(t)$.

Theorem 1. Assume $\gamma \geq 2$, and let $c: \mathbb{R} \rightarrow \mathbb{R}$ be a locally integrable T-periodic function such that, for some constants $c_{1}$ and $c_{2}$,

$$
0<c_{1} \leq c(t) \leq c_{2}, \quad \text { for a.e. } t \in \mathbb{R} .
$$

Then, for any locally integrable $T$-periodic function $e: \mathbb{R} \rightarrow \mathbb{R}$, there exists a $k_{1} \geq 1$ such that, for every integer $k \geq k_{1}$, equation (1) has a periodic solution $x_{k}(t)$ with minimal period $k T$, which makes exactly one revolution around the origin in the period time $k T$.

Theorem 1 will follow from a more general result concerning a system of the type

$$
\ddot{x}=(-h(t,|x|)+e(t)) \frac{x}{|x|} .
$$

The solutions $x(t) \in \mathbb{R}^{N}$ are functions which never attain the singularity, in the sense that

$$
x(t) \neq 0, \quad \text { for every } t \in \mathbb{R} .
$$

Since the system is radially symmetric, their orbits lie on a plane, so we will assume, without loss of generality, that $N=2$.

In Section 2 we will prove a general theorem, stated in a semi-abstract setting, which is well suited for establishing the existence of rotating solutions for such a system under a simple topological degree assumption. This assumption is verified in many different situations already considered in the literature and involves only the study of a scalar equation, the one verified by the radially oscillating solutions. Besides the periodic solutions, even in this general framework we find a whole family of quasi-periodic solutions, as in [5, 7].

As a consequence of our general theorem, in Section 3 we prove an existence result, under some assumptions which cover a complementary situation with respect to the one considered in [5, 7. Indeed, roughly speaking, in this case the nonlinearity lies below the first eigenvalue. This fact will permit us to use the classical theory of lower and upper solutions.

There is a large literature on the existence of periodic solutions for Hamiltonian systems with a singularity. In 5, we gave a brief review of the results which are more related to ours. Concerning the use of variational methods, one can see 1 and the references therein. See also [3, 11, 14, where topological methods have been employed.

Recently, the dynamics of asteroid pairs has become a topic of interest (see, e.g., [8] and the references therein), and it can also be formulated as a Keplerian type problem with a periodic force field. Another interesting topic is treated by variational methods in 2, where a periodically rotating gravitational force is considered. 


\section{A GENERAL PRINCIPLE FOR ROTATING SOLUTIONS}

In this section, we consider the system

$$
\ddot{x}=(-h(t,|x|)+e(t)) \frac{x}{|x|},
$$

where the function $e: \mathbb{R} \rightarrow \mathbb{R}$ is locally integrable and $T$-periodic and the function $h: \mathbb{R} \times] 0,+\infty\left[\rightarrow \mathbb{R}\right.$ is $T$-periodic in its first variable, as well, and $L^{1}$-Carathéodory, i.e.,

(i) $h(\cdot, r)$ is measurable and $T$-periodic, for every $r>0$;

(ii) $h(t, \cdot)$ is continuous, for almost every $t \in \mathbb{R}$;

(iii) for every compact interval $[a, b]$ in $] 0,+\infty\left[\right.$, there exists $\ell_{a, b} \in L^{1}(0, T)$ such that

$$
\left.r \in[a, b] \Rightarrow|h(t, r)| \leq \ell_{a, b}(t), \quad \text { for a.e. } t \in\right] 0, T[\text {. }
$$

We will provide a general theorem for the existence of periodic solutions of (4), whose periods are sufficiently large multiples of $T$, which rotate around the origin exactly once in their period time.

We may write the solutions of (4) in polar coordinates:

$$
x(t)=\rho(t)(\cos \varphi(t), \sin \varphi(t)),
$$

and (3) is satisfied if $\rho(t)>0$, for every $t$. Equation (44) is then equivalent to the system

$$
\left\{\begin{array}{l}
\ddot{\rho}-\frac{\mu^{2}}{\rho^{3}}+h(t, \rho)=e(t), \\
\rho^{2} \dot{\varphi}=\mu,
\end{array}\right.
$$

where $\mu$ is the (scalar) angular momentum of $x(t)$. Recall that $\mu$ is constant in time along any solution. In the following, when considering a solution of $(\mathrm{S})$, we will always implicitly assume that $\mu \geq 0$ and $\rho>0$.

We will look for solutions for which $\rho(t)$ is $T$-periodic. We thus consider the problem

$$
\left\{\begin{array}{l}
\ddot{\rho}-\frac{\mu^{2}}{\rho^{3}}+h(t, \rho)=e(t), \\
\rho(0)=\rho(T), \quad \dot{\rho}(0)=\dot{\rho}(T) .
\end{array}\right.
$$

Let $X$ be a Banach space of functions such that

$$
C^{1}([0, T]) \subseteq X \subseteq C([0, T])
$$

with continuous immersions, and set

$$
X_{+}=\{\rho \in X: \min \rho>0\} .
$$

Define the linear operator

$$
\begin{aligned}
& L: D(L) \subset X \rightarrow L^{1}(0, T), \\
& D(L)=\left\{\rho \in W^{2,1}(0, T): \rho(0)=\rho(T), \dot{\rho}(0)=\dot{\rho}(T)\right\}, \\
& L \rho=\ddot{\rho},
\end{aligned}
$$


and the Nemytzkii operator

$$
\begin{aligned}
& N_{\mu}: X_{+} \rightarrow L^{1}(0, T), \\
& \left(N_{\mu} \rho\right)(t)=\frac{\mu^{2}}{\rho^{3}(t)}-h(t, \rho(t))+e(t) .
\end{aligned}
$$

Problem $\left(P_{\mu}\right)$ is then equivalent to

$$
L \rho=N_{\mu} \rho .
$$

Taking $\sigma \in \mathbb{R}$ not belonging to the spectrum of $L$, we have that (6) can be translated to the fixed point problem

$$
\rho=(L-\sigma I)^{-1}\left(N_{\mu}-\sigma I\right) \rho .
$$

The following theorem provides a general degree assumption, only at $\mu=0$, which automatically implies, for every $\mu>0$ sufficiently small, the solvability of (S), with $\rho(t)$ a $T$-periodic function. Moreover, corresponding to an appropriate choice of $\mu$, the function $\varphi(t)-(2 \pi / k T) t$ is $k T$-periodic, for a sufficiently large integer $k$.

We will say that a set $\Omega \subseteq X$ is uniformly positively bounded below if there is a constant $\delta>0$ such that $\min \rho \geq \delta$ for every $\rho \in \Omega$.

Theorem 2. Let $\Omega$ be an open bounded subset of $X$, uniformly positively bounded below. Assume that there is no solution of (6), with $\mu=0$, on the boundary $\partial \Omega$, and that

$$
\operatorname{deg}\left(I-(L-\sigma I)^{-1}\left(N_{0}-\sigma I\right), \Omega\right) \neq 0 .
$$

Then, there exists a $k_{1} \geq 1$ such that, for every integer $k \geq k_{1}$, system (4) has a periodic solution $x_{k}(t)$ with minimal period $k T$, which makes exactly one revolution around the origin in the period time $k T$. The function $\left|x_{k}(t)\right|$ is T-periodic and, when restricted to $[0, T]$, it belongs to $\Omega$. Moreover, if $\mu_{k}$ denotes the angular momentum associated to $x_{k}(t)$, then

$$
\lim _{k \rightarrow \infty} \mu_{k}=0 .
$$

Remark 1. Condition (7) can be written, equivalently, as

$$
d_{L}\left(L-N_{0}, \Omega\right) \neq 0,
$$

where $d_{L}$ denotes the Mawhin coincidence degree; cf. [9].

Proof. Since $\Omega$ is uniformly positively bounded below, the closure of $\Omega$ is contained in $X_{+}$. We first prove that there exists a constant $M>0$ such that for every $\mu \in[0, M]$ there is no solution of (6) on the boundary $\partial \Omega$. Indeed, by contradiction, assume there are two sequences $\left(\mu_{n}\right)_{n}$ and $\left(\rho_{n}\right)_{n}$ such that $\mu_{n} \rightarrow 0, \rho_{n} \in \partial \Omega$, and

$$
\rho_{n}=(L-\sigma I)^{-1}\left(N_{\mu_{n}}-\sigma I\right) \rho_{n} .
$$

Then, $\left(\rho_{n}\right)_{n}$ and $\left(1 / \rho_{n}\right)_{n}$ are uniformly bounded, so that $\left(\left(N_{\mu_{n}}-\sigma I\right) \rho_{n}\right)_{n}$ is bounded in $L^{1}(0, T)$. Since $(L-\sigma I)^{-1}: L^{1}(0, T) \rightarrow X$ is a compact operator, there exists a subsequence, still denoted by $\left(\rho_{n}\right)_{n}$, for which $(L-\sigma I)^{-1}\left(N_{\mu_{n}}-\sigma I\right) \rho_{n}$ converges to some $\bar{\rho} \in X$. Hence $\rho_{n} \rightarrow \bar{\rho}$, as well, and, since $\partial \Omega$ is closed, $\bar{\rho} \in \partial \Omega$. Since $\rho_{n} \rightarrow \bar{\rho}$ uniformly and $\bar{\rho} \in X_{+}$, we deduce from the definition of $N_{\mu}$ that $\bar{\rho}=(L-\sigma I)^{-1}\left(N_{0}-\sigma I\right) \bar{\rho}$, so that $\bar{\rho}$ solves (6) with $\mu=0$, a contradiction to the assumptions.

By the global continuation principle of Leray-Schauder (see e.g. [15], Theorem 14.C]), there is a continuum $\mathcal{C}$ in $[0, M] \times \Omega$ connecting $\{0\} \times \Omega$ with $\{M\} \times \Omega$, 
whose elements $(\mu, \rho)$ satisfy $\left(P_{\mu}\right)$. Let us consider the function $\Phi: \mathcal{C} \rightarrow \mathbb{R}$, defined by

$$
\Phi(\mu, \rho)=\int_{0}^{T} \frac{\mu}{\rho^{2}(t)} d t .
$$

It is continuous and defined on a compact and connected domain, so its image is a compact interval. Since $\Phi(0, \rho)=0$ and $\Phi$ is not identically zero, this interval is of the type $[0, \bar{\theta}]$, for some $\bar{\theta}>0$.

We now show that, for every $\theta \in[0, \bar{\theta}]$, there are $(\mu, \rho, \varphi)$, verifying system (S), for which $(\mu, \rho) \in \mathcal{C}$, and

$$
\rho(t+T)=\rho(t), \quad \varphi(t+T)=\varphi(t)+\theta,
$$

for every $t \in \mathbb{R}$. Indeed, given $\theta \in[0, \bar{\theta}]$, there are $(\mu, \rho) \in \mathcal{C}$ such that

$$
\int_{0}^{T} \frac{\mu}{\rho^{2}(t)} d t=\theta
$$

Extending $\rho(t)$ by $T$-periodicity, the first equation in $(\mathrm{S})$ is satisfied, for every $t \in \mathbb{R}$. Moreover, defining

$$
\varphi(t)=\int_{0}^{t} \frac{\mu}{\rho^{2}(s)} d s
$$

the second equation in $(\mathrm{S})$ is also satisfied and

$$
\varphi(t+T)-\varphi(t)=\int_{t}^{t+T} \frac{\mu}{\rho^{2}(s)} d s=\int_{0}^{T} \frac{\mu}{\rho^{2}(s)} d s=\theta,
$$

for every $t \in \mathbb{R}$. Then, for every $\theta \in[0, \bar{\theta}]$, the solution of system (S) found above provides, through (5), a solution to system (4) such that

$$
x(t+T)=e^{i \theta} x(t),
$$

for every $t \in \mathbb{R}$ (for briefness we used here the complex notation).

In particular, if $\theta=\frac{2 \pi}{k}$ for some integer $k \geq 1$, then $x(t)$ is periodic with minimal period $k T$ and rotates exactly once around the origin in the period time $k T$. Hence, for every integer $k \geq 2 \pi / \bar{\theta}$, we have such a $k T$-periodic solution, which we denote by $x_{k}(t)$. Let $\left(\rho_{k}(t), \varphi_{k}(t)\right)$ be its polar coordinates, and let $\mu_{k}$ be its angular momentum. By the above construction, $\left(\mu_{k}, \rho_{k}, \varphi_{k}\right)$ verify system (S), $\left(\mu_{k}, \rho_{k}\right) \in \mathcal{C}$, and

$$
\int_{0}^{T} \frac{\mu_{k}}{\rho_{k}^{2}(t)} d t=\frac{2 \pi}{k} .
$$

Since $\rho_{k} \in \Omega$ and $\Omega$ is bounded in $C([0, T])$, there is a constant $C>0$ such that $\rho_{k}(t)<C$, for every $t \in[0, T]$. Hence,

$$
\frac{2 \pi}{k}=\int_{0}^{T} \frac{\mu_{k}}{\rho_{k}^{2}(t)} d t>T \frac{\mu_{k}}{C^{2}},
$$

so that $\lim _{k} \mu_{k}=0$. The proof is thus completed.

Remark 2. The solutions satisfying (8) are quasi-periodic with $T$-periodic radial component and with mean angular velocity, on every time interval of length $T$, equal to $\theta / T$. 


\section{An application of Theorem 2}

In this section we provide, as an application of Theorem 2, the following result, which is, in some sense, complementary to those obtained in [5, 7.

Theorem 3. Let the following two assumptions hold.

(LL) There exists a function $\eta \in L^{1}(0, T)$, with positive values, such that

$$
\begin{aligned}
& h(t, r) \geq-\eta(t), \quad \text { for every } r \in] 0,1] \text { and a.e. } t \in] 0, T[, \\
& h(t, r) \leq \eta(t), \quad \text { for every } r \geq 1 \text { and a.e. } t \in] 0, T[,
\end{aligned}
$$

and

$$
\frac{1}{T} \int_{0}^{T} \liminf _{r \rightarrow 0^{+}} h(t, r) d t>\bar{e}>\frac{1}{T} \int_{0}^{T} \limsup _{r \rightarrow+\infty} h(t, r) d t .
$$

$\left(\mathrm{H} 0_{+}\right)$There is a constant $\bar{\delta}>0$ and $a C^{1}$-function $\left.F:\right] 0, \bar{\delta}[\rightarrow \mathbb{R}$ such that

$$
\left.h(t, r) \geq F^{\prime}(r), \quad \text { for every } r \in\right] 0, \bar{\delta}[\text { and a.e. } t \in] 0, T[,
$$

and

$$
\lim _{r \rightarrow 0^{+}} F(r)=-\infty .
$$

Then, there exists a $k_{1} \geq 1$ such that, for every integer $k \geq k_{1}$, system (4) has a periodic solution $x_{k}(t)$ with minimal period $k T$, which makes exactly one revolution around the origin in the period time $k T$. Moreover, there is a constant $C>0$ such that

$$
\frac{1}{C}<\left|x_{k}(t)\right|<C, \quad \text { for every } t \in \mathbb{R} \text { and every } k \geq k_{1},
$$

and, if $\mu_{k}$ denotes the angular momentum associated to $x_{k}(t)$, then

$$
\lim _{k \rightarrow \infty} \mu_{k}=0 \text {. }
$$

Assumption (LL) is the well-known Landesman-Lazer condition, which was first introduced in [12] in the framework of elliptic partial differential equations. Condition $\left(\mathrm{H}_{+}\right)$will be needed in order to control the solutions which approach the singularity, by the use of some energy estimates. In the following, it will be convenient to extend the functions in $L^{1}(0, T)$ by $T$-periodicity to the whole line $\mathbb{R}$.

As a corollary of Theorem 3 we have that if $\gamma \geq 2$, assuming (2) and $\bar{e}>0$, the same conclusion holds for the model system (1). We thus have a proof of Theorem 1 . since the case $\bar{e} \leq 0$ has already been considered in [5, 7].

We will show that, under the assumptions of Theorem 3, taking $X=C^{1}([0, T])$, we can find an open bounded subset $\Omega$ of $X$ which satisfies the assumptions of Theorem 2

Let us first recall the following equivalent formulation of the Landesman-Lazer condition (see, e.g., [10, Proposition 4.1]).

Lemma 1. Condition (LL) is equivalent to the following.

$\left(\mathrm{LL}^{\prime}\right)$ There are a constant $d \geq 1$ and two functions $\psi_{1}, \psi_{2} \in L^{1}(0, T)$ such that

$$
\begin{aligned}
& \left.\left.\left.h(t, r) \geq \psi_{1}(t), \quad \text { for every } r \in\right] 0, \frac{1}{d}\right] \text { and a.e. } t \in\right] 0, T[\text {, } \\
& \left.h(t, r) \leq \psi_{2}(t), \quad \text { for every } r \geq d \text { and a.e. } t \in\right] 0, T[, \\
& \text { and } \\
& \frac{1}{T} \int_{0}^{T} \psi_{1}(t) d t>\bar{e}>\frac{1}{T} \int_{0}^{T} \psi_{2}(t) d t .
\end{aligned}
$$


Proof. If (LL') holds, (LL) is directly verified, since

$$
\liminf _{r \rightarrow 0^{+}} h(t, r) \geq \psi_{1}(t), \quad \limsup _{r \rightarrow+\infty} h(t, r) \leq \psi_{2}(t),
$$

for almost every $t$.

On the other hand, if (LL) holds, we have that

$$
\left.\left.\liminf _{r \rightarrow 0^{+}} h(t, r)=\lim _{n} g_{n}(t), \quad \text { with } \quad g_{n}(t)=\inf \{h(t, r): r \in] 0, \frac{1}{n}\right]\right\} .
$$

Since $\left(g_{n}\right)_{n}$ is an increasing sequence of functions, bounded from below by $-\eta$, the monotone convergence theorem implies that

$$
\lim _{n} \int_{0}^{T} g_{n}(t) d t=\int_{0}^{T} \lim _{n} g_{n}(t) d t=\int_{0}^{T} \liminf _{r \rightarrow 0^{+}} h(t, r) d t>T \bar{e} .
$$

Hence, there is a $\bar{n}$ such that $\frac{1}{T} \int_{0}^{T} g_{n}(t) d t>\bar{e}$, for every $n \geq \bar{n}$. Similarly, writing

$$
\limsup _{r \rightarrow+\infty} h(t, r)=\lim _{n} f_{n}(t), \quad \text { with } \quad f_{n}(t)=\sup \{h(t, r): r \geq n\},
$$

one finds a $\tilde{n}$ such that $\frac{1}{T} \int_{0}^{T} f_{n}(t) d t<\bar{e}$, for every $n \geq \tilde{n}$. Setting $d=\max \{\bar{n}, \tilde{n}\}$, $\psi_{1}=g_{\bar{n}}$, and $\psi_{2}=f_{\tilde{n}}$, condition (LL') follows.

Without loss of generality we assume that $e(t)$ has zero mean value, i.e.,

$$
\bar{e}=0 .
$$

Indeed, otherwise, we just replace $e(t)$ by $e(t)-\bar{e}$ and $h(t, \rho)$ by $h(t, \rho)-\bar{e}$.

Let $d \geq 1$ be as in Lemma 1. For some $\left.\left.r_{0} \in\right] 0, \frac{1}{d}\right]$, to be fixed later, we define the truncation $h_{r_{0}}: \mathbb{R} \times \mathbb{R} \rightarrow \mathbb{R}$, as follows:

$$
h_{r_{0}}(t, r)=\left\{\begin{array}{ll}
h(t, r) & \text { for } r \geq r_{0} \\
h\left(t, r_{0}\right) & \text { for } r \leq r_{0}
\end{array} .\right.
$$

This function is still $L^{1}$-Carathéodory. We consider the $T$-periodic problem

$$
\left\{\begin{array}{l}
\ddot{\rho}+h_{r_{0}}(t, \rho)=e(t) \\
\rho(0)=\rho(T), \dot{\rho}(0)=\dot{\rho}(T)
\end{array}\right.
$$

and look for a priori bounds for the solutions $\rho$, for small values of $r_{0}$. To this aim, we first show the existence of lower and upper solutions.

Lemma 2. There exist two functions $\alpha, \beta:[0, T] \rightarrow \mathbb{R}$ such that, for every $r_{0} \in$ ] $\left.0, \frac{1}{d}\right], \alpha$ is a strict lower solution of $\left(\hat{P}_{r_{0}}\right), \beta$ is a strict upper solution, and $\alpha(t) \leq 0$, $\beta(t) \geq d$, for every $t$. Moreover, every solution $\rho$ of $\left(\hat{P}_{r_{0}}\right)$ is such that $\alpha<\rho<\beta$.

Proof. Consider the functions $\psi_{1}$ and $\psi_{2}$ given by Lemma 1, Recall that they are extended to $\mathbb{R}$ by $T$-periodicity. Let $\psi_{1}(t)=\bar{\psi}_{1}+\tilde{\psi}_{1}(t)$, with $\bar{\psi}_{1}=\frac{1}{T} \int_{0}^{T} \psi_{1}(t) d t$, and similarly for $\psi_{2}$. Recall (9) and choose two $T$-periodic functions $w_{1}(t)$ and $w_{2}(t)$ such that

$$
\ddot{w}_{1}(t)=-\tilde{\psi}_{1}(t)+e(t), \quad \ddot{w}_{2}(t)=-\tilde{\psi}_{2}(t)+e(t),
$$

for almost every $t$. Set $\alpha(t)=w_{1}(t)-c$ and $\beta(t)=w_{2}(t)+c$ with $c \geq d+$ $\max \left\{\left\|w_{1}\right\|_{\infty},\left\|w_{2}\right\|_{\infty}\right\}$. Then, for every $\left.\left.r_{0} \in\right] 0, \frac{1}{d}\right], \alpha$ is a lower solution of $\left(\hat{P}_{r_{0}}\right)$, and $\beta$ is an upper solution (see [4, Theorem 7.2.1]). Hence, there is indeed a whole family of lower and upper solutions. 
Let us see that $\alpha, \beta$ are strict. Assume that $\rho$ is a solution of $\left(\hat{P}_{r_{0}}\right)$ such that $\rho(t) \geq \alpha(t)$, for every $t \in[0, T]$. Extending $\rho$ by periodicity, since $\alpha$ is also $T$ periodic, we have that $\rho(t) \geq \alpha(t)$, for every $t \in \mathbb{R}$. If by contradiction $\alpha$ is not strict, there is a $t_{0} \in \mathbb{R}$ such that $\rho\left(t_{0}\right)=\alpha\left(t_{0}\right)$. Then $\dot{\rho}\left(t_{0}\right)=\dot{\alpha}\left(t_{0}\right)$ and, for almost every $t$ in a neighborhood of $t_{0}$,

$$
\ddot{\rho}(t)-\ddot{\alpha}(t)=-h\left(t, r_{0}\right)+\psi_{1}(t)-\bar{\psi}_{1} \leq-\bar{\psi}_{1}<0,
$$

so that, integrating,

$$
\rho(t)-\alpha(t) \leq-\frac{1}{2}\left(t-t_{0}\right)^{2} \bar{\psi}_{1}
$$

and we find a contradiction. Similarly, one proves that $\beta$ is strict as well.

As shown above, once we have selected $\alpha$ and $\beta$, every function of the type $\alpha(t)-\xi$, with $\xi \geq 0$, is also a strict lower solution, and every function of the type $\beta(t)+\xi$ is a strict upper solution. This fact implies that the graphs of the solutions of $\left(\hat{P}_{r_{0}}\right)$ cannot cross any of the graphs of these lower and upper solutions. Indeed, assume by contradiction that there is a crossing of a solution $\rho(t)$ with one of the lower solutions $\alpha_{1}(t)=\alpha(t)-\xi_{1}$ above. Then, there would be a $\xi_{2} \geq \xi_{1}$ and a $t_{0} \in \mathbb{R}$ such that, setting $\alpha_{2}(t)=\alpha(t)-\xi_{2}$, one has $\rho(t) \geq \alpha_{2}(t)$ for every $t$ and $\rho\left(t_{0}\right)=\alpha_{2}\left(t_{0}\right)$, contradicting the fact that $\alpha_{2}$ is strict. The proof is then easily completed.

Remark 3. The use of lower and upper solutions for scalar equations with a singularity was first proposed by Lazer and Solimini in [13.

We now carry out the a priori bounds needed on the solutions of $\left(\hat{P}_{r_{0}}\right)$.

Lemma 3. Let $d \geq 1$ be as in Lemma 1. There exist $\left.\left.\bar{r}_{0} \in\right] 0, \frac{1}{d}\right]$ and $C>0$ such that if $\rho(t)$ is any solution of $\left(\hat{P}_{r_{0}}\right)$, with $\left.\left.r_{0} \in\right] 0, \bar{r}_{0}\right]$, then

$$
\frac{1}{C}<\rho(t)<\beta(t) \text { and }|\dot{\rho}(t)|<C \text {, for every } t \in[0, T] .
$$

Proof. The strict inequality with $\beta(t)$ is guaranteed by Lemma 2. By contradiction, assume that, for every $n \geq d$, there are $\left.\left.r_{0, n} \in\right] 0, \frac{1}{n}\right]$ and a solution $\rho_{n}(t)$ of $\left(\hat{P}_{r_{0, n}}\right)$ such that either $\min \rho_{n} \leq \frac{1}{n}$ or $\left\|\dot{\rho}_{n}\right\|_{\infty} \geq n$. For simplicity we denote by $h_{n}$ the function $h_{r_{0, n}}$.

Let us estimate the derivative of $\rho_{n}$. We know that $\rho_{n}(t)<\beta(t)$, for every $t$. Using (LL') and the Carathéodory assumption, we can find a function $\chi \in L^{1}(0, T)$ such that

$$
h_{n}\left(t, \rho_{n}(t)\right) \geq \chi(t)
$$

for almost every $t$. Since $\rho_{n}$ is $T$-periodic, for every $t$ there is a $t_{1}$ such that $t-T \leq t_{1} \leq t$, for which $\dot{\rho}_{n}\left(t_{1}\right)=\dot{\rho}_{n}\left(t_{1}+T\right)=0$. Hence,

$$
\dot{\rho}_{n}(t)=\dot{\rho}_{n}\left(t_{1}\right)+\int_{t_{1}}^{t} \ddot{\rho}_{n}=\int_{t_{1}}^{t}\left(-h_{n}\left(s, \rho_{n}(s)\right)+e(s)\right) d s \leq\|\chi\|_{1}+\|e\|_{1},
$$

while

$$
\dot{\rho}_{n}(t)=\dot{\rho}_{n}\left(t_{1}+T\right)+\int_{t_{1}+T}^{t} \ddot{\rho}_{n}=\int_{t_{1}+T}^{t}\left(-h_{n}\left(s, \rho_{n}(s)\right)+e(s)\right) d s \geq-\|\chi\|_{1}-\|e\|_{1} .
$$

Hence,

$$
\left\|\dot{\rho}_{n}\right\|_{\infty} \leq C_{1}
$$


with $C_{1}=\|\chi\|_{1}+\|e\|_{1}$. So, if $n$ is sufficiently large, we have that $\left\|\dot{\rho}_{n}\right\|_{\infty}<n$. Therefore, it has to be $\min \rho_{n} \leq \frac{1}{n}$, for $n$ large enough.

We now show that $\max \rho_{n}>\frac{1}{d}$, for every $n \geq d$. By contradiction, assume that $\max \rho_{n} \leq \frac{1}{d}$. Since $r_{0, n} \leq \frac{1}{d}$, by $\left(\mathrm{LL}^{\prime}\right)$,

$$
\ddot{\rho}_{n}(t)=-h_{n}\left(t, \rho_{n}(t)\right)+e(t) \leq-\psi_{1}(t)+e(t) .
$$

Integrating over $[0, T]$, we get a contradiction, since $\bar{e}=0$ and $\bar{\psi}_{1}>0$.

Set $\tilde{r}_{1}=\min \left\{\frac{1}{d}, \bar{\delta}\right\}$. Taking $n$ large enough, we can assume that $\tilde{r}_{1}>\frac{1}{n}$. Then there is an interval $\left[\gamma_{n}, \delta_{n}\right]$ such that

$$
\rho_{n}\left(\gamma_{n}\right)=\tilde{r}_{1}, \quad \rho_{n}\left(\delta_{n}\right)=\frac{1}{n}
$$

and

$$
\left.\frac{1}{n}<\rho_{n}(t)<\tilde{r}_{1}, \quad \text { for every } t \in\right] \gamma_{n}, \delta_{n}[.
$$

Let $\eta(t)$ be as in (LL), define

$$
\tilde{h}(t, r)=h(t, r)+\eta(t), \quad \tilde{e}(t)=e(t)+\eta(t), \quad \tilde{f}(r)=\max \left\{F^{\prime}(r), 0\right\},
$$

and let $\tilde{F}:] 0, \bar{\delta}\left[\rightarrow \mathbb{R}\right.$ be a primitive of $\tilde{f}$, i.e., $\tilde{F}^{\prime}(r)=\tilde{f}(r)$, for every $r$. Then

$$
\left.\tilde{h}(t, r) \geq \tilde{F}^{\prime}(r), \quad \text { for every } r \in\right] 0, \bar{\delta}[\text { and a.e. } t \in \mathbb{R},
$$

and

$$
\lim _{r \rightarrow 0^{+}} \tilde{F}(r)=-\infty .
$$

Recall that $r_{0, n} \leq \frac{1}{n}$. So, by (11) we have that

$$
\left.\ddot{\rho}_{n}(t)+\tilde{h}\left(t, \rho_{n}(t)\right)=\tilde{e}(t), \quad \text { for every } t \in\right] \gamma_{n}, \delta_{n}[.
$$

Let $C_{1}$ be the constant appearing in (10). Multiplying the equation in (13) by $\left(\dot{\rho}_{n}-C_{1}\right)$ and integrating on $\left[\gamma_{n}, \delta_{n}\right]$, we see that

$$
\frac{1}{2}\left[\left(\dot{\rho}_{n}-C_{1}\right)^{2}\right]_{\gamma_{n}}^{\delta_{n}}+\int_{\gamma_{n}}^{\delta_{n}} \tilde{h}\left(t, \rho_{n}(t)\right)\left(\dot{\rho}_{n}(t)-C_{1}\right) d t=\int_{\gamma_{n}}^{\delta_{n}} \tilde{e}(t)\left(\dot{\rho}_{n}(t)-C_{1}\right) d t .
$$

Hence, by (10), there is a constant $c_{2}>0$ for which

$$
\left|\int_{\gamma_{n}}^{\delta_{n}} \tilde{h}\left(t, \rho_{n}(t)\right)\left(\dot{\rho}_{n}(t)-C_{1}\right) d t\right| \leq c_{2},
$$

for every $n$. On the other hand, since $\dot{\rho}_{n}-C_{1} \leq 0$,

$$
\begin{aligned}
\int_{\gamma_{n}}^{\delta_{n}} \tilde{h}\left(t, \rho_{n}(t)\right)\left(\dot{\rho}_{n}(t)-C_{1}\right) d t & \leq \int_{\gamma_{n}}^{\delta_{n}} \tilde{F}^{\prime}\left(\rho_{n}(t)\right)\left(\dot{\rho}_{n}(t)-C_{1}\right) d t \\
& \leq \int_{\gamma_{n}}^{\delta_{n}} \tilde{F}^{\prime}\left(\rho_{n}(t)\right) \dot{\rho}_{n}(t) d t \\
& =\left[\tilde{F}\left(\rho_{n}(t)\right)\right]_{\gamma_{n}}^{\delta_{n}} \\
& \leq \tilde{F}\left(\frac{1}{n}\right)-\tilde{F}\left(\tilde{r}_{1}\right) .
\end{aligned}
$$

Using (12), when $n$ tends to infinity, we get a contradiction to (14), thus ending the proof of the lemma. 
We now take $\bar{r}_{0}>0$ and $C>0$ as given by Lemma 3 . We can also assume that $\beta(t) \leq C$, for every $t$. Let us fix $r_{0}=\min \left\{\bar{r}_{0}, \frac{1}{C}\right\}$. As a consequence of Lemma 3 , any solution $\rho(t)$ of $\left(\hat{P}_{r_{0}}\right)$ is also a solution of $\left(P_{0}\right)$. Define $\Omega$ to be the following open and bounded subset of $C^{1}([0, T])$ :

$$
\Omega=\left\{\rho \in C^{1}([0, T]): \frac{1}{C}<\rho(t)<\beta(t) \text { and }|\dot{\rho}(t)|<C \text {, for every } t \in[0, T]\right\} .
$$

Clearly, $\Omega$ is uniformly positively bounded below.

In order to apply Theorem 2, we need to verify assumption (7), i.e., that the topological degree is not zero. Let us define, for problem $\left(\hat{P}_{r_{0}}\right)$, the Nemytzkii operator

$$
\begin{aligned}
& \hat{N}: C^{1}([0, T]) \rightarrow L^{1}(0, T), \\
& (\hat{N} \rho)(t)=-h_{r_{0}}(t, \rho(t))+e(t) .
\end{aligned}
$$

By the classical theory on lower and upper solutions, we have that, setting

$$
\hat{\Omega}=\left\{\rho \in C^{1}([0, T]): \alpha(t)<\rho(t)<\beta(t) \text { and }|\dot{\rho}(t)|<C \text {, for every } t \in[0, T]\right\},
$$

it is

$$
\operatorname{deg}\left(I-(L-\sigma I)^{-1}(\hat{N}-\sigma I), \hat{\Omega}\right)=1
$$

see, e.g., 4, Theorem 3.1.12]. As $\Omega$ is a subset of $\hat{\Omega}$ and, by Lemma 3 , every solution of $\left(\hat{P}_{r_{0}}\right)$ is in $\Omega$, the excision property of the degree implies that

$$
\operatorname{deg}\left(I-(L-\sigma I)^{-1}(\hat{N}-\sigma I), \hat{\Omega}\right)=\operatorname{deg}\left(I-(L-\sigma I)^{-1}(\hat{N}-\sigma I), \Omega\right) .
$$

Since $\hat{N}$ and $N_{0}$ coincide on $\Omega$, we finally have

$$
\operatorname{deg}\left(I-(L-\sigma I)^{-1}\left(N_{0}-\sigma I\right), \Omega\right)=1 .
$$

The proof of Theorem 3 is thus completed.

\section{REFERENCES}

[1] A. Ambrosetti and V. Coti Zelati, Periodic solutions of singular Lagrangian systems, Progress in Nonlinear Differential Equations and their Applications 10, Birkhäuser Boston, Inc., Boston, MA, 1993. MR.1267225 (95b:58054)

[2] K. Chen, Variational constructions for some satellite orbits in periodic gravitational force fields, Amer. J. Math. 132 (2010), 681-709. MR2666904

[3] J. Chu and D. Franco, Non-collision periodic solutions of second order singular dynamical systems, J. Math. Anal. Appl. 344 (2008), 898-905. MR.2426318 (2010d:37115)

[4] C. De Coster and P. Habets, Two-Point Boundary Value Problems, Lower and Upper Solutions, Elsevier, Amsterdam, 2006. MR 2225284 (2007k:34001)

[5] A. Fonda and R. Toader, Periodic orbits of radially symmetric Keplerian-like systems: a topological degree approach, J. Differential Equations 244 (2008), 3235-3264. MR2420520 (2009b:34127)

[6] A. Fonda and R. Toader, Periodic orbits of radially symmetric systems with a singularity: the repulsive case, Adv. Nonlinear Stud., to appear.

[7] A. Fonda and A. Ureña, Periodic, subharmonic, and quasi-periodic oscillations under the action of a central force, Discrete Contin. Dyn. Syst. 29 (2011), 169-192. MR.2725286

[8] F. Gabern, W. S. Koon, J. E. Marsden and D. J. Scheeres, Binary asteroid observation orbits from a global dynamical perspective, SIAM J. Appl. Dyn. Syst. 5 (2006), 252-279. MR2237147 (2007d:70018)

[9] R.E. Gaines and J. Mawhin, Coincidence Degree and Nonlinear Differential Equations, Lect. Notes Math. 568, Springer, Berlin, 1977. MR0637067 (58:30551)

[10] J.-P. Gossez and P. Omari, Non-ordered lower and upper solutions in semilinear elliptic problems, Comm. Partial Differential Equations 19 (1994), 1163-1184. MR.1284805 (96b:35047) 
[11] P. Habets and L. Sanchez, Periodic solutions of dissipative dynamical systems with singular potentials, Differential Integral Equations 3 (1990), 1139-1149. MR1073063 (92a:34040)

[12] E. M. Landesman and A. C. Lazer, Nonlinear perturbations of linear elliptic boundary value problems at resonance, J. Math. Mech. 19 (1970), 609-623. MR0267269 (42:2171)

[13] A. C. Lazer and S. Solimini, On periodic solutions of nonlinear differential equations with singularities, Proc. Amer. Math. Soc. 99 (1987), 109-114. MR866438 (87k:34064)

[14] P. J. Torres, Non-collision periodic solutions of forced dynamical systems with weak singularities. Discrete Contin. Dyn. Syst. 11 (2004), 693-698. MR2083439 (2005e:34133)

[15] E. Zeidler, Nonlinear Functional Analysis and Its Applications, Vol. 1, Springer, New YorkHeidelberg, 1986. MR816732 (87f:47083)

Dipartimento di Matematica e Informatica, Università di Trieste, Piazzale Europa 1, I-34127 Trieste, ItAly

E-mail address: a.fonda@units.it

Dipartimento di Ingegneria Civile e Architettura, Università di Udine, Via delle SCIENZE 208, I-33100 Udine, Italy

E-mail address: toader@uniud.it 